ISSN: 2215-2644

revedu@gmail.com

Universidad de Costa Rica

Costa Rica

\title{
Profesorado de educación primaria con mención en Chile: entre lo planteado en los estándares orientadores para egresados de pedagogía y lo real de la práctica docente
}

Sánchez Bustos, Mario Arturo; Torres Díaz, Romi Alejandra

Profesorado de educación primaria con mención en Chile: entre lo planteado en los estándares orientadores para egresados de pedagogía y lo real de la práctica docente

Revista Educación, vol. 45, núm. 2, 2021

Universidad de Costa Rica, Costa Rica

Disponible en: https://www.redalyc.org/articulo.oa?id=44066178013

DOI: https://doi.org/10.15517/revedu.v45i1.42928

\section{(c) 1 (1) 9}

Esta obra está bajo una Licencia Creative Commons Atribución-NoComercial-SinDerivar 3.0 Internacional. 


\title{
Profesorado de educación primaria con mención en Chile: entre lo planteado en los estándares orientadores para egresados de pedagogía y lo real de la práctica docente
}

\author{
Primary Education Professors in Chile: A Comparison of Standard Guidelines for Education Graduates with Real- \\ Life Teaching Practices
}

Mario Arturo Sánchez Bustos

Universidad Católica del Maule, Chile

msanchezb@ucm.cl

(iD) https://orcid.org/0000-0003-4632-5399

Romi Alejandra Torres Díaz

Universidad Católica del Maule, Chile

ratorres@ucm.cl

(D) https://orcid.org/0000-0002-8700-6856
DOI: https://doi.org/10.15517/revedu.v45i1.42928

Redalyc: https://www.redalyc.org/articulo.oa? $\mathrm{id}=44066178013$
Recepción: 25 Julio 2020

Aprobación: 24 Octubre 2020

\section{Resumen:}

En el contexto de un Sistema Nacional de Aseguramiento de Calidad en educación, que exige precisión en la definición de los aprendizajes del currículo nacional; el presente estudio compara lo planteado en los estándares orientadores para personas egresadas de carreras de pedagogía en educación básica con lo que ocurre en las escuelas de primaria; ya que se asume que para cada asignatura se designarán profesionales con preparación en el área. Este artículo pretende contribuir a la reflexión sobre la formación docente y la necesidad de potenciar el trabajo en todas las disciplinas para generar aprendizajes más contextualizados y significativos, y cómo esta situación presenta una buena oportunidad para que las universidades puedan crear una especialización, post título o mención para docentes del primer ciclo básico en las áreas indicadas sin preparación. Para esta investigación se trabajó con 50 docentes y 30 directivos de escuelas subvencionadas; además, se revisó la oferta académica de 28 universidades que dictan esta carrera en Chile. Los resultados evidencian que no existe una relación entre la práctica y la formación profesional en una mención específica, porque en realidad las y los docentes imparten clases también en otras disciplinas como Artes Visuales, Música o Tecnología, independiente si recibieron o no preparación en ellas, y en consecuencia, al no tener certeza del dominio que posee el profesorado sobre estas áreas del conocimiento, no se puede garantizar que la formación que reciban sus estudiantes sea de calidad, lo que demanda que las escuelas formadoras de docentes pongan un acento especial en la necesidad de impartir menciones en todas las asignaturas impuestas como obligatorias.

Palabras clave: Calidad de la educación, Formación de profesores de primaria, Enfoque interdisciplinario, Plan de estudios universitarios, Política educacional.

\section{Abstract:}

In order to gauge the national teacher-training curriculum as required by Chile's Education Quality Assurance Agency, this study compares the guiding standards established for primary school education graduates with the inhouse experience of actual elementary schools teachers. This article reflects on the need for enhanced efforts to make learning more multidisciplinary, contextualized and meaningful under the assumption that teachers with training in a specific subject area are hired for that corresponding job. This would serve as a good opportunity for universities to establish graduate or postgraduate degrees for primary school education teachers in areas where there are gaps. The study enrolled 50 teachers and 30 principals from subsidized schools. At the same time, teacher training programs at 28 Chilean universities were reviewed with an emphasis on primary school education. Based on the results, however, it is difficult to compare teacher practice and their professional training based on specific subject areas, since, they are required to be malleable and teach classes in disciplines such as Art, Music or Technology, while on the job, regardless of their prior preparation for those subjects. Furthermore, teacher mastery of these subjects cannot be ensured since teacher-training does not address these aspects. As a result, there is no way of verifying if students are receiving a quality education in these subject areas; yet, universities could address this gap by emphasizing the need to address all compulsory subject areas in their teacher-training curriculum. 
KEYWORDS: Educational Quality, Primary School Teacher Training, Interdisciplinary Approach, University, College Curriculum, Educational Policies.

\section{INTRODUCCIÓN}

En Chile, en enero de 2011, fue aprobada La Ley de Calidad y Equidad de la Educación, la cual centra su interés en avanzar hacia un mejor y más justo sistema educativo. A partir de esto se busca contar con profesorado cada vez mejor formado, que sean profundos conocedores de las disciplinas y de sus estrategias de enseñanza, así como de los aspectos pedagógicos que debe dominar para enfrentar la realidad del aula. (Ministerio de Educación [MINEDUC], 2012).

La Formación General en la Educación Básica de Chile considera sectores de aprendizaje obligatorios: Lenguaje y Comunicación; Lengua Indígena (obligatorio ofrecerlo en los establecimientos con alta densidad de matrícula indígena); Idioma Extranjero (obligatorio desde $5^{\circ}$ a $8^{\circ}$ básico); Matemática; Ciencias Naturales; Historia, Geografía y Ciencias Sociales; Tecnología; Artes Visuales; Música; Educación Física; Orientación y Religión (obligatorio ofrecerlo, pero opcional cursarlo para alumnos y alumnas) (MINEDUC, 2009).

Un establecimiento de educación básica debe tener el personal docente idóneo que sea necesario y que le permita cumplir con las funciones que le corresponden, entendiéndose por docente idóneo/a a la/el que cuente con el título de profesional de la educación del respectivo nivel y especialidad cuando corresponda, o esté habilitado/a para ejercer la función docente según las normas legales vigentes (Ley 20370, 2009).

Las instituciones de educación superior, dedicadas a la formación inicial de profesorado de primaria, cuentan con estándares entregados por el Ministerio de Educación; dichos estándares, son una referencia para quienes forman docentes, puesto que trasparentan los conocimientos, habilidades y competencias que deben trabajar con las y los estudiantes en formación (MINEDUC, 2009).

En los Estándares orientadores para egresados/as de carreras de pedagogía en educación básica, se asume que el/la nuevo/a profesor/a generalista enseñará, fundamentalmente, en cuatro áreas disciplinarias: Lenguaje y Comunicación; Matemática; Historia, Geografía y Ciencias Sociales; y Ciencias Naturales; y se espera que en las otras áreas de aprendizaje, de manera preferente, puedan enseñar profesorado de Educación Básica con mención en dicha área, o profesorado de Educación Media que fueron autorizados/as para desempeñarse en otros niveles (MINEDUC, 2012).

Sin embargo, actualmente no existe una norma que establezca requisitos específicos para las/los docentes que impartan asignaturas como Educación Artística (Artes Visuales, Música y Artes Escénicas en algunos casos), Educación Física, Idioma Extranjero y Tecnología, es la/el Director del establecimiento educacional quien establece cuál es el/la docente idóneo/a para su colegio, dependiendo de la asignatura que imparta.

$\mathrm{Al}$ revisar diversas investigaciones sobre el tema, estas dan cuenta que, al dar prioridad sólo a cuatro asignaturas, se evidencia la disminución de las asignaturas y conocimientos artísticos como una consecuencia, lo que impacta en la educación integral de las y los estudiantes (Orbeta y Oyanedel, 2018). Además, muestran que en un sistema educativo que se presenta como innovador y avanzado, no se entiende que las manifestaciones artísticas queden prácticamente fuera del mapa curricular básico, desoyendo a instituciones nacionales e internacionales que abogan por reforzar los estudios artísticos desde edades tempranas (López, 2018).

El presente trabajo expone las menciones obtenidas por profesorado de educación primaria de la región del Maule, comparándolas con las asignaturas que estos y estas han impartido en su práctica docente, considerando los criterios para ser asignados/as a ellas. Se presenta también, la oferta académica vigente de universidades que imparten la carrera de pedagogía básica, enfocándose en las menciones que allí se pueden obtener. 
Se busca reflexionar sobre las asignaturas en las cuales no se cuenta con una/un profesor especialista, dado que, para un buen desarrollo de estas, es necesario un conocimiento específico en cada una de ellas, según lo propuesto por el MINEDUC (2012) y también por diversos autores especialistas en las áreas en cuestión. Contrastándolo con la oferta académica, para analizar la especialización en estas asignaturas del futuro profesorado en el corto plazo y los posibles impactos de esta realidad, sobre lo que se espera en materia de calidad y equidad de la educación.

\section{Dominio en disciplinas del currículum}

En el sistema educativo chileno, se denominó Bases Curriculares al instrumento principal del currículum nacional, y aquí se encuentra definido lo que se espera que los alumnos aprendan (MINEDUC, 2018).

De acuerdo a lo planteado por Beijaard, Verloop y Vermunt (2000) y por Rodríguez, Saavedra y Castillo (2015), la calidad de un sistema educativo depende en gran medida del grado de manejo que tiene una/ un profesor sobre los contenidos de la disciplina que imparte y la profundidad con la que los aborda, la comprensión de los conceptos principales y la relación entre ellos.

Los planes y programas de estudio que se encuentran alineados con las Bases curriculares, entregando herramientas para el desarrollo integral del estudiante, en la práctica contrastan con un currículum estructurado que valida algunas asignaturas sobre otras, potenciando aquellas relacionadas con el conocimiento productivo y económico (Hernández, 2012).

$\mathrm{Al}$ considerar las asignaturas afines con lo económico como lo más importante, la formación escolar no está destinada al consumo, sino a la formación integral de la persona; queda expuesta a la decisión de cada comunidad escolar para decidir qué hacer con ella, ya que estos saberes no se miden en las pruebas estandarizadas ni se expresan en indicadores de calidad de los sistemas educativos, relegándolas así a un segundo plano (Belletich, Wilhelmi y Angel, 2016).

Respecto de la educación artística, y específicamente la educación en las artes visuales, esta tiene un papel de gran relevancia en la etapa de la enseñanza básica, pues se espera que, mediante ella, las y los alumnos se inicien en la comprensión de este legado de la humanidad y que, al apreciarlo, enriquezcan sus posibilidades de imaginar, simbolizar y crear (MINEDUC, 2018).

Es complejo establecer un diálogo y una discusión sobre la importancia de las artes en la educación (Quintana, 2016), enseñar arte en las escuelas es alfabetizar estéticamente al sujeto (Rojas, 2017), la educación artística desarrolla la sensibilidad, estimula la capacidad creadora del individuo, potenciando otros modos de sentir, pensar, actuar y experimentar. Para Elichiry y Regatky (2010), es una acción extremadamente importante, sobre todo si se pone el énfasis en la experiencia individual de cada alumno/a (Fernández, 2019).

Sin embargo, las prácticas pedagógicas desarrolladas en el subsector de Educación Artística se caracterizan por una desvalorización de esta; lo que emana desde los planes y programas de estudio (Hernández, 2012).

Por su parte, las universidades responsables de la formación inicial docente, han descuidado el currículum de la Educación Artística, se observa que el profesorado de educación básica no posee una especialización o formación universitaria en esta área (Pérez y Llona, 2011) y, a pesar de las discusiones académicas internas, ha prevalecido un marcado desinterés o total ausencia por incorporar esta disciplina en las mallas de las carreras de educación primaria, junto con una permanente falta de oportunidades de formación continua para las y los docentes en servicio, más aún en las regiones alejadas de la capital del país (Cárdenas, Lagos y Figueroa, 2016).

La mayoría de las y los estudiantes y egresados/as de las carreras de pedagogía primaria, no se sienten preparados/as para enseñar las áreas artísticas en el contexto escolar. Eso responde principalmente a que la formación está focalizada en otras áreas (Orbeta y Oyanadel, 2018).

Respecto de la asignatura de tecnología, se plantea que uno de sus objetivos es desarrollar el pensamiento creativo y divergente, buscar soluciones alternativas a problemas ya resueltos, con la finalidad de optimizar e innovar productos tecnológicos para que cumplan mejor su propósito (MINEDUC, 2018). 
En esta asignatura es fundamental que el y la estudiante observe su entorno, determine problemas y pueda proponerles soluciones, para esto, es preciso transformar los sistemas educativos y lograr que el profesorado se convierta en líderes del cambio, o lo que es lo mismo, profesorado emprendedor, para desarrollar competencias directamente relacionadas con el emprendimiento, como son la creatividad, la innovación o el liderazgo, para luego poder generar más empleo y lograr un mayor crecimiento económico y social (Arruti y Paños, 2019).

A pesar de los argumentos que tiene a su favor, la educación tecnológica no ha alcanzado todavía una posición sólida, en general, quienes se encargan de la alfabetización tecnológica comparten concepciones distorsionadas y empobrecidas de la tecnología, lo que obstaculiza una enseñanza capaz de favorecer una inmersión en la cultura tecnocientífica, generando poco interés y no beneficiando el aprendizaje (Ferreira, Vilches y Gil, 2012).

En lo que respecta a las clases de artes musicales, en estas se debe escuchar, interpretar, crear, comentar, analizar e investigar música. Se debe comprender la música desde su totalidad, integrando el lenguaje musical y sus elementos: las cualidades musicales del sonido, el silencio, la melodía, el ritmo, la textura, entre otros; y procedimientos como reiteraciones, contrastes, patrones, variaciones, con su propósito expresivo (MINEDUC, 2018).

Interpretar un instrumento y todo lo que en él se involucra con un resultado satisfactorio es una actividad que implica bastante tiempo, dedicación y mucha disciplina (Fuertes, 2019). Hay una escasez de profesorado idóneo en la música, presentan vacíos técnicos; la actividad generalmente es entregada al profesorado primario o de otras asignaturas que demuestran ciertas habilidades, entusiasmo y amor por el canto colectivo (Alarcón, 2001), quienes no cuentan con la formación necesaria (Valverde y Godall, 2018), por ello, es muy importante que los programas universitarios se ajusten al currículo nacional chileno. Es decir, la formación inicial del profesorado de primaria se debería orientar hacia la formación especializada en educación musical, lo que implica dominar tanto competencias musicales como pedagógicas para llevar a cabo la didáctica de la música (Angel, 2018).

En la sociedad actual, escuchar o crear son competencias importantes, y la música

incide en trabajar estos aspectos, sin embargo, goza de escasa atención y valoración (Casals, Carrillo y González, 2014); a este sector de la educación no se le asigna la importancia necesaria (Lorenzo y Ruxandra, 2016); incluso las y los alumnos de educación primaria le restan importancia a esta asignatura (Lorite, 2018).

La concepción de la música en la escuela tiene un discurso un tanto ambiguo; por una parte, se habla de los beneficios de su presencia y aprendizaje, pero en la práctica es poco promovida desde las instituciones escolares. Muchas veces es vista como un complemento de relleno (Montoya, 2016), quizás se deba a la pérdida de un sentido que articule la función de la música en la escuela junto con su finalidad social visible (Nahuelán, 2019).

Como se ha dicho, actualmente, la formación docente en Chile se basa en los Estándares orientadores para egresados/as de pedagogía, lo que en general se ha demostrado es que, cuando los estándares son impuestos como medida de control, no han generado un mejoramiento educativo. Sin embargo, cuando estos son definidos por el profesorado o las universidades, se obtienen mejores resultados (Zuzovsky y Libman, 2006). Los estándares chilenos son una medida de orientación y control, pero sólo abordan algunas asignaturas, lo que provoca inquietud en el profesorado en formación, ya que necesitan que sea su formador/a quien entregue orientaciones sobre la práctica pedagógica real (Rodríguez, 2004; Vaillant, 2009). Se muestra una gran preocupación por la aritmética y la lectoescritura, olvidando otras competencias fundamentales en la vida profesional (Fullan, 2018).

En síntesis, en el currículum nacional chileno hay asignaturas para las cuales existen indicadores de calidad bien definidos, que son aquellas asociadas al conocimiento productivo y, por otro lado, asignaturas que promueven el desarrollo integral de la persona, las cuales no se miden en pruebas estandarizadas; esto provoca 
una despreocupación por estas áreas del conocimiento; a pesar de la relevancia demostrada de cada una de ellas en la formación escolar y de las competencias específicas necesarias para impartirlas.

\section{Metodología}

Esta investigación cualitativa permite comparar las menciones obtenidas por el profesorado de educación primaria durante su formación profesional con las asignaturas que estos imparten en su práctica docente; considerando los criterios para esta asignación, se utilizaron las siguientes técnicas para la recolección de datos:

Se realizaron entrevistas estructuradas a participantes voluntarios/as, con las siguientes preguntas estructurales y para ejemplificar, expuestas en la Tabla 1 y Tabla 2 ya que, de acuerdo con lo planteado por Hernández, Fernández y Baptista (2014), es frecuente este tipo de muestreo en investigaciones con las características mencionadas,en donde el interés es que todas las personas entrevistadas respondan a las mismas preguntas, entregando datos específicos, para luego compararlos y obtener conclusiones.

TABLA 1

Preguntas de la entrevista realizada a docentes

Pregunta
¿Obtuvo alguna mención en su formación
profesional?
¿Cuántas horas de clases realiza en la asignatura
de la mención obtenida y cuántas horas tiene por
contrato en el colegio? (durante el año 2019).
¿Qué otras asignaturas ha tenido que impartir
durante su práctica docente en años anteriores?
¿Qué aspectos cree usted que influyen para
establecer la carga horaria de las asignaturas
distintas a Lenguaje, Matemática, Historia y
Ciencias Naturales en su establecimiento?

\author{
Proposito \\ Identificar caracteristicas de su formación \\ profesional y si está relacionada con las \\ asignaturas impartidas. \\ Identificar la porción del tiempo destinada a \\ hacer clase en la mención obtenida en su \\ formación profesional. \\ Identificar las asignaturas impartidas a lo largo \\ de su vida profesional, y si están relacionadas \\ con su formación profesional. \\ Identificar criterios para establecer la carga \\ horaria de las asignaturas distintas a Lenguaje, \\ Matemática, Historia y Ciencias Naturales.
}

Fuente: Elaboración Propia

TABLA 2

Pregunta realizada a directivos/as

$\begin{array}{ll}\text { Pregunta } & \text { Propósito } \\ \text { ¿Qué aspectos considera para decidir la carga } & \text { Identificar criterios para establecer la carga } \\ \text { horaria de las asignaturas distintas a Lenguaje, } & \text { horaria de las asignaturas distintas a } \\ \text { Matemática, Historia y Ciencias Naturales en su } & \text { Lenguaje, Matemática, Historia y Ciencias } \\ \text { establecimiento? } & \text { Naturales. }\end{array}$

Fuente: Elaboración Propia

Se realizaron entrevistas durante el año 2019 para ser analizadas en profundidad, sin pretender generalizar los resultados, a 28 docentes ( 18 mujeres y 10 hombres) que ejercen en educación primaria en cuatro colegios de la provincia de Curicó y a 22 docentes ( 14 mujeres y 8 hombres) pertenecientes también a cuatro colegios de la ciudad de Talca, siendo todos colegios que reciben financiamiento del estado.

Se entrevistó también a 30 directivos/as de las mismas instituciones ( 23 hombres y 7 mujeres), con quienes se trianguló la información entregada por las y los docentes.

Las personas entrevistadas fueron informadas de los objetivos del estudio cuando se solicitó su ayuda antes de realizar las preguntas, y respondieron a estas sin límite de tiempo. 
Para poder entregarles sentido a las respuestas obtenidas, estas se organizaron de la siguiente forma:

Para comenzar, las respuestas del profesorado se clasificaron de acuerdo con las asignaturas realizadas durante su práctica pedagógica, con la finalidad de identificar aquellas que sean comúnmente impartidas por el profesorado de primaria y aquellas que no son usualmente entregadas por ellos y ellas; y, además, se contempló la mención obtenida por cada uno y una en su formación profesional, cuando aplicaba, considerando la porción de su carga académica destinada a ella.

Para esto, se elaboró la siguiente tabla:

TABLA 3

Tabulación de las respuestas de las personas docentes

Asignaturas imparticas

Entrevistado/a Leng. Y Com. Idioma Ext. Matemática C. Naturales Hist., Geo. Y Cs. Tecnologia Artes Visuales Música Ed. Fisica Mención Horas destinadas a mención cantidad/total

Fuente: Elaboración Propia

En la Tabla 3 se marcaron con una $\mathrm{X}$ las asignaturas impartidas por cada una de las personas docentes durante su práctica pedagógica; en la penúltima columna, se anotó la mención obtenida, indicando que no aplica [NA], para aquellas que no obtuvieron mención en su formación profesional y en la última columna se indicó la porción del horario destinado a trabajar en la asignatura de la mención obtenida, marcando 0 para quienes no tienen.

Luego, para identificar los criterios para establecer la carga horaria en las asignaturas distintas a Lenguaje, Matemática, Historia y Ciencias Naturales, las respuestas recopiladas se analizaron mediante una codificación abierta para poder organizarlas en categorías, buscando conceptos, temas y patrones presentes en los datos, a fin de otorgarles sentido, interpretarlos y explicarlos en función del planteamiento del problema (Hernández et al., 2014; Cohen y Gómez, 2019), resaltando así ideas referentes a cuatro áreas: experiencias previas exitosas, completación de horario, iniciativa y/o entusiasmo de/la profesor/a y formación académica. Este análisis permitió elaborar la siguiente tabla:

TABLA 4

Tabulación de las respuestas asociadas a la asignación de carga

\begin{tabular}{lllll} 
& \multicolumn{3}{c}{ Aspectos considerados en la asignación de carga } \\
& \multicolumn{3}{c}{ Iniciativa } \\
Entrevistado/a & $\begin{array}{l}\text { Experiencias } \\
\text { previas }\end{array}$ & $\begin{array}{l}\text { Completación } \\
\text { y/o }\end{array}$ & Formación \\
& exitosas & de horario & $\begin{array}{l}\text { entusiasmo } \\
\text { del }\end{array}$ & académica \\
& & & profesorado &
\end{tabular}

Fuente: Elaboración Propia

Posteriormente, se revisaron nuevamente cada una de las entrevistas, para ser tabuladas de acuerdo con los criterios presentes en la Tabla 4, marcando con una $\mathrm{X}$ todos los aspectos mencionados en las respuestas recopiladas.

La Tabla 5 muestra la tabulación realizada a las respuestas de un docente y un directivo entrevistados: 
TABLA 5

Tabulación de las respuestas asociadas a la asignación de carga de un docente y directivo

Entrevistado Respuesta

Directivo

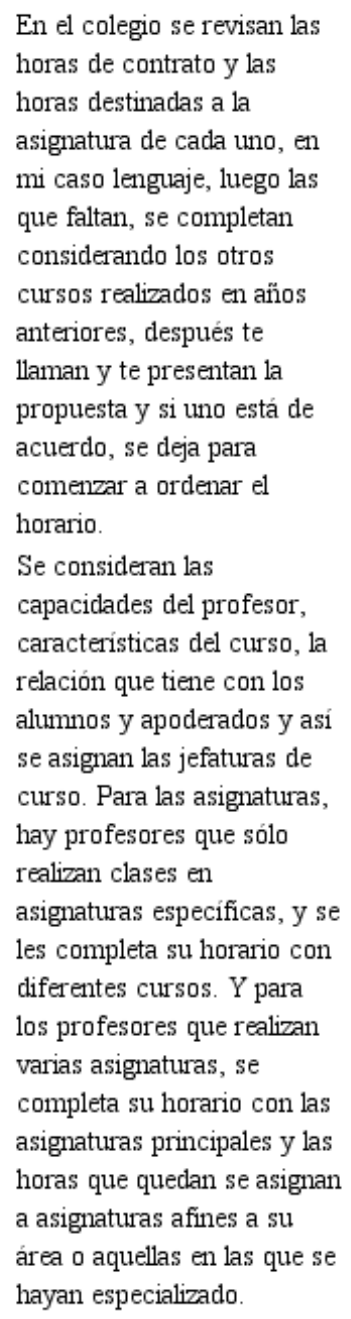

Fuente: Elaboración Propia

Las respuestas de los entrevistados se clasificaron considerando los lineamientos predominantes, ya que no siempre las respuestas tenían una postura radical. Sin embargo, en todas hubo tendencias que prevalecían.

Se consideraron también 28 universidades que durante el año 2020 imparten la carrera de pedagogía básica en Chile, tanto públicas como privadas, y estas se clasificaron de acuerdo a las menciones que ofrecían en la carrera, incluyendo aquellas que no entregan mención. Lo que se pretende con esta revisión es visualizar la especialización del futuro profesorado de primaria que egrese en los próximos años en la región del Maule en áreas distintas a Lenguaje y Comunicación; Matemática; Historia, Geografía y Ciencias Sociales; y Ciencias Naturales, y compararlo con lo que ocurre en instituciones de otras regiones, que pudiesen presentar una realidad distinta a la exhibida en este estudio. Para esto se elaboró la siguiente tabla: 
TABLA 6

Tabulación de las menciones ofrecidas por las carreras de pedagogía básica

\begin{tabular}{|c|c|c|c|c|c|c|c|c|c|c|}
\hline \multirow{4}{*}{ Universidades } & \multicolumn{4}{|c|}{ Menciones } & & & & & \\
\hline & & & & & Hist., & & & & & \\
\hline & $\begin{array}{l}\text { Leng. } \\
Y \\
\text { Com. }\end{array}$ & $\begin{array}{l}\text { Idioma } \\
\text { Ext. }\end{array}$ & Matemática & $\begin{array}{l}\text { C. } \\
\text { Naturales }\end{array}$ & $\begin{array}{l}\text { Geo. } \\
\text { Y }\end{array}$ & Tecnología & $\begin{array}{l}\text { Artes } \\
\text { Visuales }\end{array}$ & Música & $\begin{array}{l}\text { Ed. } \\
\text { Física }\end{array}$ & $\begin{array}{l}\text { Sin } \\
\text { Mención }\end{array}$ \\
\hline & & & & & Cs. & & & & & \\
\hline
\end{tabular}

Fuente: Elaboración Propia

En la Tabla 6 se marcaron con una $X$ todas las casillas de acuerdo a las menciones que ofrecía la universidad, para aquellas instituciones que impartían más de una en su carrera de pedagogía básica.

En el intento de cumplir con los objetivos planteados para este estudio, se generó un elemento innovador, que es un aporte de esta investigación a la educación primaria del país en relación a otros artículos citados, que abordan este tema desde la perspectiva de su área del conocimiento en particular; este trabajo, en cambio, muestra un análisis conjunto de las asignaturas, lo que permite realizar una triangulación entre:

a) La formación profesional de los docentes en ejercicios: que muestra la especialización de las personas docentes a través de las menciones obtenidas.

b) Las asignaturas impartidas por las personas docentes: que muestra las asignaturas que han impartido, teniendo o no la formación profesional para realizarlas

c) La oferta académica vigente de las universidades del país: que dirige la formación y de alguna forma influencia la práctica pedagógica de futuras personas docentes en al menos los próximos 4 años.

\section{Resultados}

Los resultados de las entrevistas muestran que el 58\% del profesorado entrevistado había obtenido una mención en su formación profesional inicial, de este, el $31 \%$ se especializó en Lenguaje y Comunicación, un $31 \%$ en Matemática, un $21 \%$ en Ciencias Naturales y un 17\% en Historia, Geografía y Ciencias Sociales y $0 \%$ en otras asignaturas.

Respecto a la mención obtenida en la formación profesional, en promedio, el profesorado destina el 36\% de su carga horaria a realizar clases en la asignatura en la cual obtuvieron su mención.

Por otra parte, del total de personal docente entrevistado, el 6\% imparte clases solo de Matemática, que coincide con la asignatura que tiene su mención; el 100\% ha tenido que impartir clases de Matemática en alguna oportunidad, el $94 \%$ ha tenido que impartir clases de Lenguaje y Comunicación, el $90 \%$ ha realizado clases de Ciencias Naturales y el $88 \%$ lo ha hecho de Historia, Geografía y Ciencias Sociales.

En otras asignaturas, el 84\% de las personas docentes había impartido en alguna oportunidad clases de Tecnología, el 76\% clases de Música y el 70\% clase de Artes Visuales. Ninguna persona docente de educación primaria entrevistada ha realizado clases de Religión, Inglés o Educación Física.

Respecto de las consideraciones para la asignación de carga horaria en asignaturas distintas a Lenguaje, Matemática, Ciencias Naturales e Historia y Geografía y Ciencias Sociales, la información obtenida, tanto de parte de las personas docentes como de las y los directivos, se muestra de forma conjunta en la siguiente figura: 


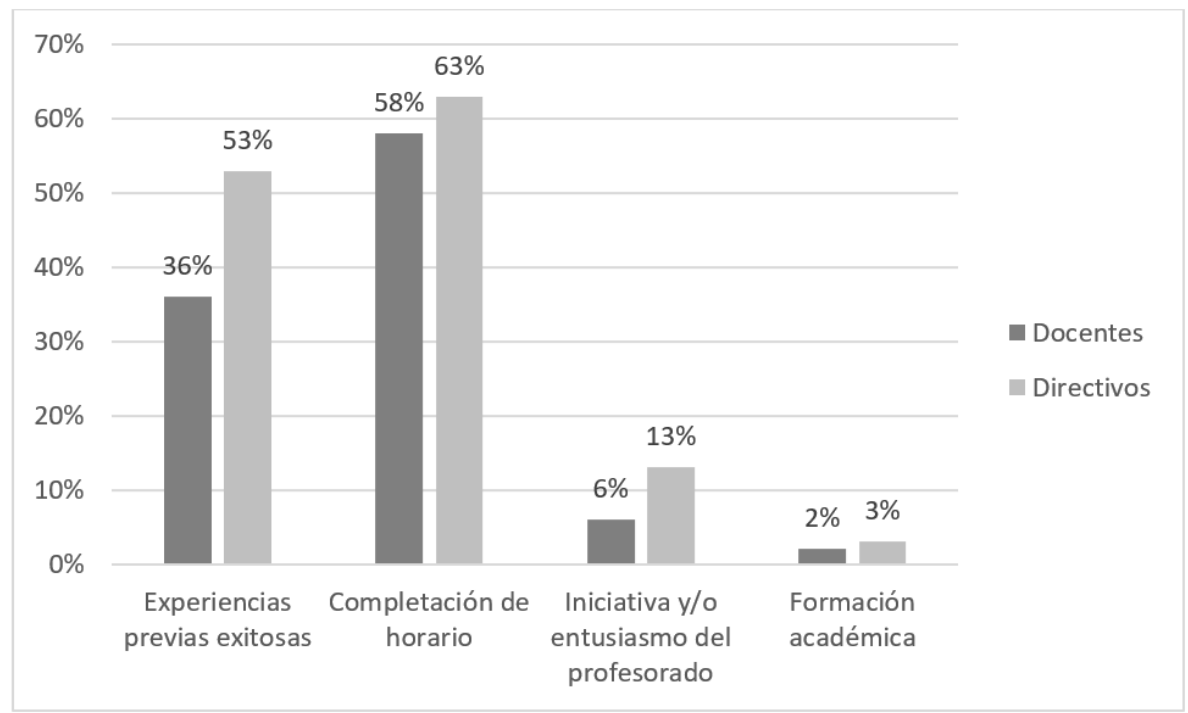

FIGURA 1.

Distribución de los aspectos a considerar para la asignación de carga horaria al personal docente en asignaturas distintas a Lenguaje, Matemática, Ciencias Naturales e Historia y Geografía y Ciencias Sociales Fuente: Elaboración propia

La Figura 1 muestra que, del personal docente, el 36\% menciona en su respuesta las experiencias previas exitosas, el $58 \%$ la completación de horario, el $6 \%$ a petición de ellos y ellas mismas y el $2 \%$ alude a la formación académica; mientras que, de las personas directivas entrevistadas, el $53 \%$ menciona las experiencias previas exitosas, el 63\% hace alusión a la completación de horario, el 13\% menciona el entusiasmo del propio docente por impartir la asignatura y el 3\% habla de la formación académica.

Respecto de la oferta académica, en la región del Maule hay 3 universidades que imparten la carrera de pedagogía básica y todas ellas ofrecen una mención a sus estudiantes; el 67\% imparte las menciones de Matemática y Lenguaje; el 33\% imparte las menciones en Ciencias Naturales, Historia y Geografía, y Ciencias Sociales, el 33\% imparte la mención en Inglés. Cabe destacar que en las universidades aludidas sólo se imparten las menciones indicadas.

Respecto de la oferta académica de las 28 universidades que imparten la carrera a nivel nacional, el $21 \%$ de ellas no ofrece mención específica a sus estudiantes; del $79 \%$ de instituciones que sí imparte una especialización a sus estudiantes de pedagogía primaria, el 86\% imparte la mención de Matemática, el 82\% mención Lenguaje, 50\% en Ciencias Naturales, 50\% en Historia y Geografía y Ciencias Sociales, el 27\% en Inglés y el 5\% en Artes. Es importante mencionar que ninguna universidad imparte una mención en Tecnología o Música.

\section{DiscuSIÓN}

Los resultados de las entrevistas muestran que hay un $42 \%$ de las personas docentes que no obtuvieron mención durante su formación inicial, por lo tanto, no se tiene una medición del dominio técnico de las disciplinas que imparten a sus estudiantes y que, de acuerdo con lo planteado por Beijaard, Verloop y Vermunt (2000) y por Rodríguez, Saavedra y Castillo (2015), es muy importante en materia de calidad de educación.

Por otra parte, para las y los docentes que sí obtuvieron mención en su formación profesional, esta no tiene relación con las asignaturas impartidas en escuelas de primaria de la región del Maule, ya que sólo el 31 \% de ellos y ellas tiene su mención en matemática y el 100\% de ellos y ellas ha tenido que impartir esta asignatura 
en alguna oportunidad, lo que implica que el $69 \%$ lo ha hecho sin tener un conocimiento medible en dicha disciplina, lo mismo ocurre con el 63\%, que ha tenido que impartir Lenguaje, el 69\% lo ha hecho en Ciencias Naturales y el $71 \%$ en Historia y Geografía y Ciencias Sociales, sin tener mención en estas asignaturas, lo que dista de lo propuesto por el Ministerio de Educación en sus estándares orientadores para egresados/as de carreras de pedagogía en educación básica (MINEDUC, 2012), y por diversos autores/as que exponen la importancia de contar con personas profesionales especializadas para impartir estas asignaturas (Cárdenas y Troncoso, 2014; Pérez y Llona, 2011; Arruti y Paños, 2019; Valverde y Godall, 2018).

La misma situación ocurre en otras asignaturas, la mayoría del profesorado entrevistado ha realizado clases de Tecnología (84\%), Música (76\%) y Artes Visuales (70\%), sin considerar una preparación profesional en ellas, en contraste con lo propuesto por diversos/as autores/as que resaltan la importancia de contar con un/a profesor/a conocedor/a de la disciplina (Cárdenas y Troncoso, 2014; Casals et al., 2014; Ferreira et al., 2012; Hernández, 2012; López, 2018; Pérez y Llona, 2011; Lorenzo y Ruxandra, 2016; Valverde y Godall, 2018).

Se puede apreciar, a partir de la información entregada en entrevistas, que Inglés, Religión y Educación Física son impartidas por docentes con título o especialización en ellas, lo que sí se condice, con lo supuesto por los estándares orientadores del Ministerio de Educación.

A partir de la información recopilada, en relación con los aspectos a considerar para la asignación de carga horaria a los y las docentes en asignaturas distintas a Lenguaje, Matemática, Ciencias Naturales e Historia y Geografía y Ciencias Sociales, tanto en lo mencionado por docentes como directivos/as, prevalece la completación de la carga horaria como la razón principal, relegando como el aspecto con menos consideración la formación académica de quienes imparten estas asignaturas, en contraste con lo propuesto por el MINEDUC (2012), lo que concuerda con la nula preparación en estas áreas en su formación inicial, del profesorado entrevistado.

Respecto a la información recopilada de las universidades de la región del Maule que imparten pedagogía en primaria y sus menciones, se desprende que al menos en los próximos 4 años no egresarán en la región, docentes con menciones ni en artes ni música ni en tecnología; y si se considera la oferta académica vigente a nivel nacional, de ninguna de las universidades consideradas egresarán, al menos durante los próximos 4 años, profesionales con mención ni en tecnología ni en música, de lo cual se puede deducir que la calidad y equidad de la enseñanza en educación básica integral es incierta; ya que la formación que recibe el profesorado de primaria, en las asignaturas distintas a las cuatro llamadas fundamentales, depende de la importancia otorgada por sus respectivas casas de estudio; al no existir estándares reguladores en ellas, los resultados muestran que las universidades del país no han dado importancia a las menciones que no están reguladas por los estándares de aprendizajes, en particular no se ofrece una mención en tecnología ni en música, a pesar del importante impacto que tienen estas áreas en el desarrollo y progreso del país (Arruti y Paños, 2019).

\section{CoNCLUSIón}

A partir del estudio realizado, se concluye que atendiendo a los estándares emanados del Ministerio de Educación, éstos pueden ser acogidos literalmente por las universidades formadoras de docentes, y poner énfasis o preocuparse solo de las cuatro asignaturas, llamadas fundamentales, descuidando aspectos relevantes que entregan otras disciplinas, que aportan a la formación, y ayudan a desarrollar integralmente a la persona, desatendiendo con ello una visión generalista, que les permita manejar los diversos aspectos culturales y disciplinares.

Dentro de este estudio, y respondiendo a los objetivos de investigación, se establece que las asignaturas desatendidas son principalmente artes visuales, música y educación tecnológica; ya que estas, en general, son impartidas por docentes sin una preparación profesional en la disciplina, lo que no garantiza una educación de calidad como lo exige la Ley 20370 (2009) y difiere con lo propuesto por el MINEDUC (2012) en los estándares orientadores para egresados/as de pedagogía. 
De la triangulación realizada, con la información recolectada, se concluye que el profesorado en ejercicio imparte asignaturas en las cuales no tiene formación académica específica para realizarlas, y que esta situación no cambiará en al menos los próximos 4 años, ya que las instituciones formadoras no están preparando a docentes en las áreas con déficit de profesionales especializados.

Dentro de los impactos de esta realidad, en el corto plazo no se podrá considerar la formación académica de los y las docentes como uno de los criterios predominantes al momento de asignar la carga horaria y en consecuencia al no tener certeza del dominio que posee el/la docente sobre la disciplina que imparte, no se puede garantizar que la formación que reciban sus estudiantes sea de calidad.

\section{RECOMENDACIONES}

Se sugiere que las escuelas formadoras de docentes pongan un acento especial en la necesidad de impartir menciones en todas las asignaturas impuestas como obligatorias por el MINEDUC (2009).

Considerando las asignaturas que no cuentan con profesorado especializado, se recomienda realizar un cambio con los y las docentes en ejercicio, mediante perfeccionamientos, talleres, seminarios, entre otros; y con los y las estudiantes en formación en las universidades, mediante nuevas prácticas pedagógicas; con la finalidad de llevar a cabo un cambio concreto en el corto/mediano plazo, que les permita tener una formación sólida en las asignaturas que impartirán, para así aportar a la formación integral, equitativa y de calidad a sus estudiantes.

Para escuelas de instituciones formadoras de docentes primarios/as, o para quienes imparten programas de magíster, se presenta como una buena oportunidad para crear una especialización, post título o mención para docentes de primer ciclo básico en las áreas que no están siendo atendidas con una mención.

Como reflexión final, se plantea que la búsqueda de una educación integral de calidad es un compromiso profesional y ético de cada una de las instituciones educativas y debería separarse de las pruebas estandarizadas, las cuales miden principalmente las cuatro asignaturas fundamentales, y lo hacen de la misma forma para todos y todas las estudiantes del país, cuyas realidades son múltiples y diversas.

\section{REFERENCIAS BIBLIOGRÁFICAS}

Alarcón, V. (2001). Crecer Cantando: una visión hacia el futuro de la música coral en Chile. Revista musical chilena, 55(195). doi: http://dx.doi.org/10.4067/S0716-27902001019500007

Angel, R. (2018) Formación inicial del profesorado generalista en Chile: Análisis desde la perspectiva de la educación musical. Revista de Docencia Universitaria, 16(1), 87-101. Recuperado de https://dialnet.unirioja.es/servlet/ar ticulo? codigo $=6511334$

Arruti, A. y Paños, J. (2019). Análisis de las menciones del grado en Educación Primaria desde la perspectiva de la competencia emprendedora. Revista Complutense de Educación, 30(1), 17-33. Recuperado de https://revistas.u cm.es/index.php/RCED/article/download/55448/4564456548996

Beijaard, D., Verloop, N. y Vermunt, J. (2000). Teachers' Perceptions of Professional Identity: An Exploratory Study From a Personal Knowledge Perspective. Teaching and teacher education, 16(7), 749-764.

Belletich, O. Wilhelmi, M. y Angel, R. (2016). La educación musical en la formación básica en España. El problema de la dispersión curricular. Revista Perspectiva Educacional, 55(2), 158-170. Recuperado de http://www.perspe ctivaeducacional.cl/index.php/peducacional/article/view/454/216

Cárdenas, R., Lagos, I. y Figueroa, E. (2016). El profesorado de educación básica y su contribución a la enseñanza de las artes visuales en la escuela. Arte, Individuo y Sociedad, 28(3), 475-493. Recuperado de https://www.redalyc .org/pdf/5135/513554410006.pdf 
Cárdenas, R. y Troncoso, A. (2014). Importancia de las artes visuales en la educación: un desafío para la formación docente. Revista Electrónica Educare, 18(3), 191-201. Recuperado de https://www.scielo.sa.cr/pdf/ree/v18n3/ a11v18n3.pdf

Casals, A., Carrillo, C. y González, C. (2014) La música también cuenta: combinando matemáticas y música en el aula. Revista Electrónica de Música en la Educación, 34,1-17. Recuperado de https://www.researchgate.net/publicati on/268978033_La_musica_tambien_cuenta_combinando_matematicas_y_musica_en_el_aula

Cohen, N. y Gómez, G. (2019). Metodología de la investigación, ¿Para qué? La producción de los datos y los diseños. Buenos Aires: Teseo.

Elichiry, N. y Regatky, M. (2010). Aproximación a la educación artística en la escuela. Anuario de Investigaciones, 17, 129-134. Recuperado de https://www.redalyc.org/pdf/3691/369139946072.pdf

Fernández, H. (2019) La enseñanza de artes visuales en los PCN-Arte. Revista Profissäo docente, 19(42), 01-15. Recuperado de http://www.revistas.uniube.br/index.php/rpd/article/view/1325/1445

Ferreira, C., Vilches, A. y Gil, D. (2012). Concepciones docentes acerca de la naturaleza de la tecnología y de las relaciones Ciencia, Tecnología, Sociedad y Ambiente en la educación tecnológica. Enseñanza de las Ciencias: revista de investigación y experiencias didácticas, 30(2), 197-218. Recuperado de https://www.raco.cat/index.ph $\mathrm{p} /$ ensenanza/article/view/254510

Fuertes, D. (2019). Criterios de formación básica en música para la admisión a programas de pregrado en Bogotá. (Tesis de maestría). Universidad Pedagógica Nacional de Colombia, Bogotá.Recuperado de http:// hdl.handle.net/20.500.12209/11378

Fullan, M. (2018) The principalship has changed 2020 here we come! Principal Connections, 22, 18-19.

Hernández, A. (2012). La retirada de las artes en la escuela. Revista de Estudios y Experiencias Pedagógicas, 11(21), 109-117. Recuperado de http://www.redalyc.org/pdf/2431/243124125007.pdf

Hernández, R.; Fernández, C. y Baptista, M. (2014). Metodología de la investigación. México D.F: Mcgraw-hill interamericana editores.

Ley 20370 (12 de septiembre de 2009). Ley general de educación, Diario Oficial de la República de Chile. Recuperado de https://www.leychile.cl/Navegar?idNorma $=1006043$

López, N. (2018) Educación musical y currículo en la enseñanza primaria española: de la legislación general a la concreción autonómica. Revista da Abem, 26(41), 56-76. Recuperado de http://www.abemeducacaomusical.co $\mathrm{m} . \mathrm{br} /$ revistas/revistaabem/index.php/revistaabem/article/view/782

Lorenzo, O. y Ruxandra, I. (2016). Percepciones sobre el entorno profesional del profesorado de Enseñanzas Musicales Superiores en Rumanía. Dedica. Revista de educação e humanidades, 9, 193-206. Recuperado de https://revista seug.ugr.es/index.php/dedica/article/view/6889/6001

Lorite, M. (2018). Las prácticas del arte sonoro motivan por encima de otras prácticas a trabajar la música. Afluir, 1, 32-51. Recuperado de https://www.afluir.es/index.php/afluir/article/view/5

Ministerio de Educación (2009). Objetivos Fundamentales y Contenidos Mínimos Obligatorios de la Educación Básica y Media Actualización 2009. Santiago: Él mismo. Recuperado de https://bit.ly/3tmvhzo

Ministerio de Educación (2012). Estándares orientadores para egresados de carreras de pedagogía en educación básica, estándares pedagógicos y disciplinarios. Santiago: LOM Ediciones Ltda. Recuperado de https://www.cpeip.cl/wp -content/uploads/2019/03/Est\%C3\%A1ndares_B\%C3\%A1sica.pdf

Ministerio de Educación (2018). Bases Curriculares Primero a Sexto Básico Santiago Primera Edición. Santiago: Ministerio de Educación. Recuperado de https://curriculumnacional.mineduc.cl/614/articles-22394_bases.pdf

Montoya, J. (2016) La musicología como ancla de la educación musical: del historicismo al audiovisual. La albolafia: revista de humanidades y cultura, 9, 61-74. Recuperado de https://dialnet.unirioja.es/servlet/articulo?codigo= 5769270

Orbeta, A. y Oyanedel, R. (2018). En vías de desaparición. Antecedentes para entender la disminución de las artes en la formación inicial docente de educación primaria en Chile. Arte, individuo y sociedad, 30(2), 375-394. Recuperado de https://revistas.ucm.es/index.php/ARIS/article/viewFile/57622/4564456546933 
Nahuelán, O. (2019) La música en el contexto escolar chileno: estudio exploratorio desde la perspectiva del profesorado. (Tesis de maestría). Universidad Autonoma de Barcelona Recuperado de http:// repositorio.conicyt.cl/handle/10533/241477

Pérez, V. y Llona, E. (2011). Estudio sobre el Estado Actual de la Educación Artística en la Región Metropolitana. IDIE de Fomento y Fortalecimiento de la Educación Artística. Santiago: Idie. Recuperado de https://mediacionartistic a.files.wordpress.com/2014/03/educacion_artistica_chile.pdf

Quintana, P. (2016). Interdisciplina, cultura visual y educación artística en Chile: de la prueba estandarizada a la invisibilización de las artes en la escuela. Paideia Surcolombiana, 21(21), 61-69. doi: https://doi.org/10.25054 /01240307.1465

Rodríguez, N. (2004). Retos de la formación de docentes en Venezuela. Revista de Pedagogía, 25(73), 03-12.

Rodríguez, C., Saavedra, R. y Castillo, V. (2015) Expectativa, Cobertura y Dominio Curricular: Percepciones del profesorado en la enseñanza de la Matemática Revista Paradigma, 36(2), 177-201.

Rojas, D. (2017) Alfabetización Visual una Estrategia Pedagógica para los procesos de enseñanza y Aprendizaje de las artes Plásticas. (Tesis de maestría).Fundación Universitaria Los Libertadores, Bogotá, Colombia Recuperado de https://repository.libertadores.edu.co/handle/11371/1197? show=full

Vaillant, D. (2009). Formación de profesores de Educación Secundaria: realidades y discursos, Revista de Educación, $350,105-122$.

Valverde, H. y Godall, P. (2018) Música Tradicional en el Aula: Aportaciones para un Aprendizaje Significativo en la Escuela. Revista Electrónica de LEEME, 41, 16-34. Recuperado de https://ojs.uv.es/index.php/LEEME/arti cle/view/10530/11863

Zuzovsky, R. y Libman, Z. (2006) Standards of Teaching and Teaching Test: Is the Right Way to Go? Studies in Educational Evaluation, 32, 37-52. doi: https://doi.org/10.1016/j.stueduc.2006.01.006

\section{INFORMACIÓN ADICIONAL}

Cómo citar: Sánchez Bustos, M.A., Torres Díaz, R.A. (2021). Profesorado de educación primaria con mención en Chile: entre lo planteado en los estándares orientadores para egresados de pedagogía y lo real de la práctica docente. Revista Educación, 45(2). Recuperado de http://doi.org/10.15517/revedu.v45i1.42928 